Article

\title{
GLUL Ablation Can Confer Drug Resistance to Cancer Cells via a Malate-Aspartate Shuttle-Mediated Mechanism
}

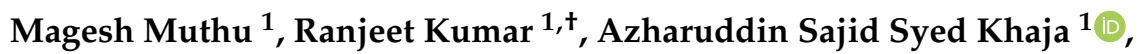 \\ Jonathan D. Gilthorpe ${ }^{2}{ }^{(D}$, Jenny L. Persson ${ }^{1}$ and Anders Nordström ${ }^{1, *}$ \\ 1 Department of Molecular Biology, Umeå University, 90187 Umeå, Sweden; magesh.muthu@umu.se (M.M.); \\ ranjeetmku09@gmail.com (R.K.); sk.azharuddin@umu.se (A.S.S.K.); jenny.persson@umu.se (J.L.P.) \\ 2 Department of Pharmacology and Clinical Neuroscience, Umeå University, 90187 Umeå, Sweden; \\ jonathan.gilthorpe@umu.se \\ * Correspondence: anders.nordstrom@umu.se; Tel.: +46-90-785-25-61; Fax: +46-90-77-26-30 \\ + Present address: Public Health Research Institute at New Jersey Medical School, Rutgers Biomedical and \\ Health Sciences, Rutgers, The State University of New Jersey, Newark, NJ 07103, USA.
}

Received: 13 November 2019; Accepted: 3 December 2019; Published: 5 December 2019

\begin{abstract}
Glutamate-ammonia ligase (GLUL) is important for acid-base homeostasis, ammonia detoxification, cell signaling, and proliferation. Here, we reported that GLUL ablation conferred resistance to several anticancer drugs in specific cancer cell lines while leaving other cell lines non-resistant to the same drugs. To understand the biochemical mechanics supporting this drug resistance, we compared drug-resistant GLUL knockout (KO) A549 non-small-cell lung carcinoma (NSCLC) cells with non-resistant GLUL KO H1299 NSCLC cells and found that the resistant A549 cells, to a larger extent, depended on exogenous glucose for proliferation. As GLUL activity is linked to the tricarboxylic acid (TCA) cycle via reversed glutaminolysis, we probed carbon flux through both glycolysis and TCA pathways by means of ${ }^{13} \mathrm{C} 5$ glutamine, ${ }^{13} \mathrm{C} 5$ glutamate, and ${ }^{13} \mathrm{C} 6$ glucose tracing. We observed increased labeling of malate and aspartate in A549 GLUL KO cells, whereas the non-resistant GLUL KO H1299 cells displayed decreased ${ }^{13} \mathrm{C}$-labeling. The malate and aspartate shuttle supported cellular NADH production and was associated with cellular metabolic fitness. Inhibition of the malate-aspartate shuttle with aminooxyacetic acid significantly impacted upon cell viability with an $\mathrm{IC}_{50}$ of $11.5 \mu \mathrm{M}$ in resistant GLUL KO A549 cells compared to $28 \mu \mathrm{M}$ in control A549 cells, linking resistance to the malate-aspartate shuttle. Additionally, rescuing GLUL expression in A549 KO cells increased drug sensitivity. We proposed a novel metabolic mechanism in cancer drug resistance where the increased capacity of the malate-aspartate shuttle increased metabolic fitness, thereby facilitating cancer cells to escape drug pressure.
\end{abstract}

Keywords: GLUL; NSCLC; drug resistance; metabolomics; glutamine; glycolysis; metabolism; targeted metabolomics; LC-MS

\section{Introduction}

Cancer cells undergo a process of continuous clonal evolution, which increases the chances of selecting for drug resistance during therapy [1-3]. Although recent therapeutic advances have improved the specificity of cancer treatment, drug resistance is an ongoing and serious problem for patient outcome [4]. Resistance mechanisms can manifest as alterations in the drug target, activation of pro-survival pathways, or ineffective induction of cell death. However, there is no drug available to target resistance mechanisms, per se. Overexpression of the multidrug resistance gene $(A B C B 1)$, encoding the permeability glycoprotein P-gp has been attributed to ineffective treatment [5-7] and 
directly implicated in treatment failure [8-10]. Targeting P-gp with a number of novel inhibitors has not yet translated into clinical utility due to toxicity and pharmacokinetic complications [11]. Identification of novel mechanisms that support cancer cell survival under therapeutic pressure is important in order to find ways of overcoming drug resistance, which ultimately results in lethality.

While deregulated cellular metabolism appears to be an emerging hallmark of cancer drug resistance [12-15], deregulated energetics involving alterations in cellular metabolism to support neoplastic proliferation is a fundamental hallmark of cancer [16]. Central to this metabolic deregulation is Warburg's discovery of aerobic glycolysis, where glucose is converted to lactate even when oxygen supply is sufficient to support oxidative phosphorylation. However, several studies have shown that aerobic glycolysis alone is insufficient to support the core metabolism required for cancer cell proliferation $[17,18]$. The tricarboxylic acid (TCA) cycle is a metabolic intersection that connects cellular energy metabolism with more distant metabolic pathways through key nodes for anaplerosis/cataplerosis. One such node is $\alpha$-ketoglutaric acid, which can be supplied through a glutaminolysis mechanism, whereby glutamine is first converted to glutamate by the action of glutaminase (GLS). Glutamate conversion, for example, by glutamate dehydrogenase (GLUD) generates $\alpha$-ketoglutaric acid. It is well known that many cancer cell types are dependent on increased flux from glutamine to glutamate and $\alpha$-ketoglutaric acid [19]. Hence, upregulated glutamine metabolism is an attractive target for cancer therapy, as evidenced by an ongoing trial with the glutaminolysis inhibitor CB-839 (ClinicalTrials.gov Identifier: NCT03428217 and NCT02771626).

Glutamate-ammonia ligase (GLUL) catalyzes the ATP-dependent synthesis of glutamine from glutamate and ammonia, which is the reverse of the initial glutaminolysis step. GLUL plays an important role in glutamate and ammonia detoxification and acid-base homeostasis. GLUL was shown to be involved in pathological cell migration during angiogenesis [20]. Thus, GLUL could be important beyond its main catalytic activity during tumor growth [20]. Increased GLUL expression has been reported as an early marker of hepatocellular carcinoma [21], promoting breast cancer cell proliferation [22], and also found to be an unfavorable prognostic marker in patients with glioblastoma multiforme (GBM) and ovarian cancer $[23,24]$. Our group has previously identified reduced GLUL transcription to be associated with resistance to the chemotherapeutic agent daunorubicin in clones of acute lymphoblastic leukemia (ALL) [14]. This finding prompted us to examine if a targeted reduction of GLUL expression could induce drug resistance. We investigated the effect of reduced GLUL expression using siRNA or lentiviral CRISPR-Cas9 mediated knockout (KO), as well as doxycycline-inducible shRNA-mediated knockdown (KD) in different cancer cell lines. Interestingly, GLUL KO/KD resulted in a gain of function phenotype with induced drug resistance in specific cancer cell types, including the non-small cell lung cancer (NSCLC) cell line A549. Metabolic profiling and stable isotope-labeled tracer experiments showed that resistance was supported through increased glucose dependence coupled with increased activity in the malate-aspartate shuttle, which is a mechanism for transporting electrons into mitochondria and thus fueling regeneration of NADH from NAD+. The activity of the malate-aspartate shuttle has been associated with longevity in yeast [25] and supports up to $20 \%$ of the respiration rate in various tumor types [26]. Here, we demonstrated that pharmacological inhibition of the malate-aspartate shuttle reduced viability in resistant GLUL KO A549 cells compared to control cells, thus connecting malate-aspartate metabolism with drug tolerance in cancer cells. Furthermore, re-expression of GLUL in KO cells restored the sensitivity of cells to drug treatment, suggesting that the expression level of GLUL might influence drug sensitivity in specific cancer cell types. Since the genetic loss of function of catalytic enzymes rarely results in a gain of function phenotype, our data suggested that the level of GLUL expression could fine-tune metabolic fitness, which in turn might offer therapeutic opportunities for combination therapies targeting metabolic fitness during induction treatment in order to suppress selection of resistant clones. 


\section{Results}

\subsection{Transient GLUL Knockdown Induces Drug Resistance}

We previously observed that drug-resistant ALL cells lacked GLUL transcription [14]. In the present study, we explored whether or not reduced GLUL expression resulted in drug resistance in solid tumor-derived cell lines. We examined GLUL protein levels by western blotting in a panel of cancer cell lines, including A549, H1299, H460 (NSCLC), HeLa (cervical cancer), HCC1954 (breast ductal carcinoma), MDA-MB-231 (triple-negative breast cancer-TNBC). A relatively high level of GLUL expression was found in HeLa cells compared to the other lines (Figure 1A). To test whether GLUL KD could induce drug resistance, we first evaluated the effectiveness of siRNA-mediated GLUL KD by western blot analysis. After $72 \mathrm{~h}$ of siRNA transfection, there was a profound decrease in GLUL protein expression in all of the cell lines tested (Figure 1B). Cells were then treated with the chemotherapeutic agent docetaxel (20 or $30 \mathrm{nM}$ for $72 \mathrm{~h}$ ), and the cell viability was assessed by MTS assay. Interestingly, knocking down GLUL promoted drug resistance in two of the cell lines (A549 and HCC1954; Figure 1C). As GLUL KD induced the highest level of drug resistance in A549 cells but had no apparent effect in the NSCLC H1299 cells, we chose to compare these two cell lines further to identify potential resistance mechanisms.

\subsection{GLUL Knockout Cells Display Drug Resistance}

We next used a lentiviral CRISPR/Cas9 system to generate complete and stable GLUL KOs in NSCLC A549 and H1299 cells. These cell lines constitute a good experiment/control pair as they are both of NSCLC origin while demonstrating resistance/non-resistance phenotype upon GLUL KO. The approaches yielded clones that were devoid of GLUL protein expression (Supplementary Figure S1A). Two weeks after transduction, independent clones were selected and propagated. For A549, we utilized clones 1 and 4 for further investigation (Figure 2A, Supplementary Figure S2A). GLUL KO A549 and control lines were exposed to various chemotherapeutic agents, including dasatinib, pazopanib, imatinib, docetaxel, and cisplatin. Cell viability was then determined by means of MTS assay. Exposure to each of the compounds in a dose-dependent manner resulted in a significant loss of viability in control A549 cells, and $\mathrm{IC}_{50}$ values more than doubled in A549 GLUL KO cells (Figure 2B, Supplementary Figure S2A). To characterize the broad spectrum of drug resistance in GLUL KO A549 cells further, we performed clonogenic assays. Cells were grown in the presence of dasatinib $(5 \mu \mathrm{M})$, pazopanib $(10 \mu \mathrm{M})$, imatinib $(10 \mu \mathrm{M})$, cisplatin $(5 \mu \mathrm{M})$, and docetaxel $(3 \mathrm{nM})$ for 12 days. Control cells displayed a reduced ability for colony formation when compared to the GLUL KOs (Figure 2C, Supplementary Figure S2B), further demonstrating enhanced drug resistance in the GLUL KO cells. 
A
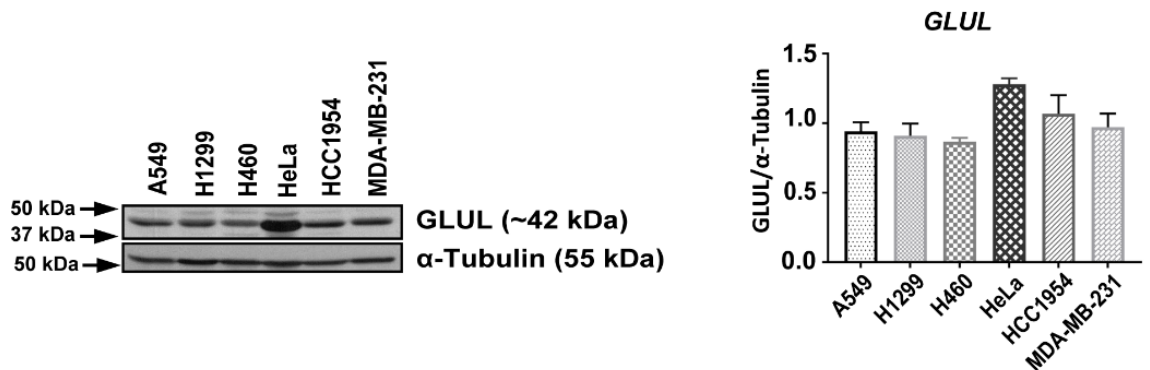

B
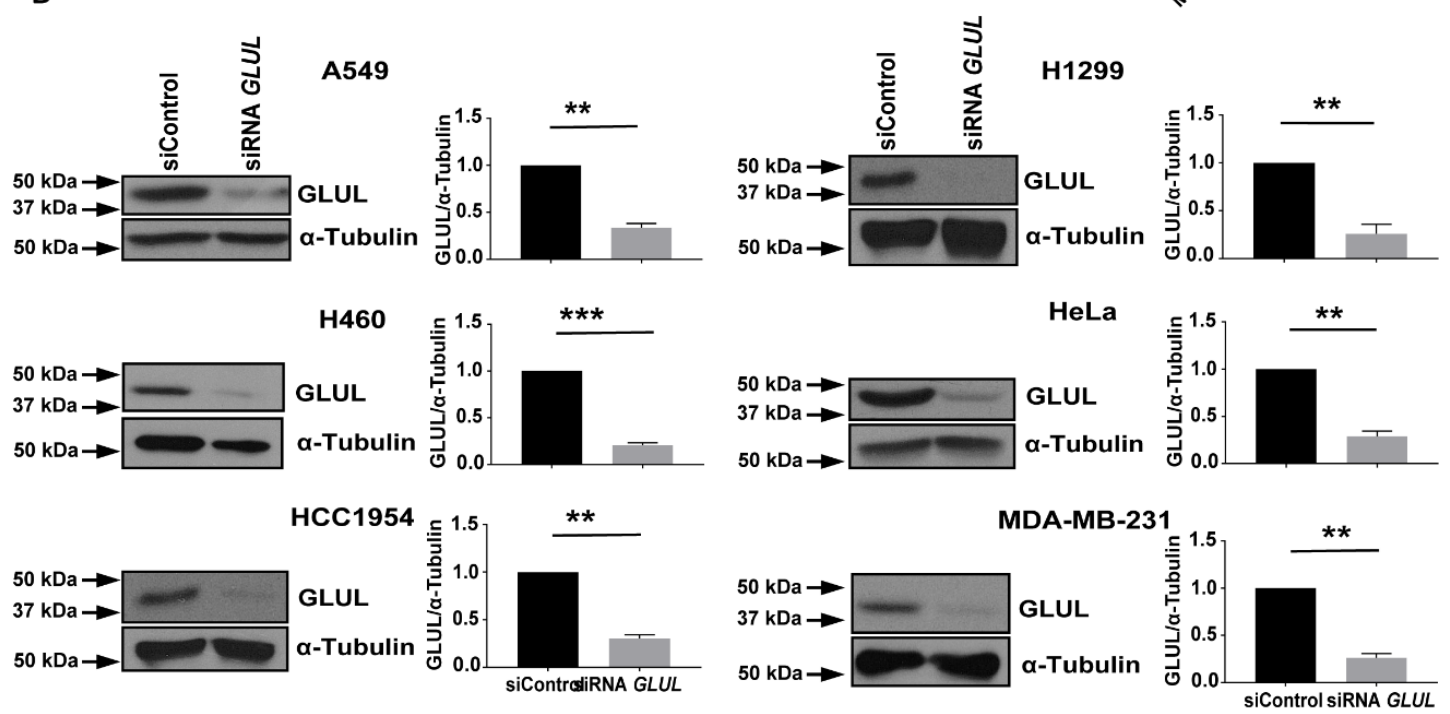

C
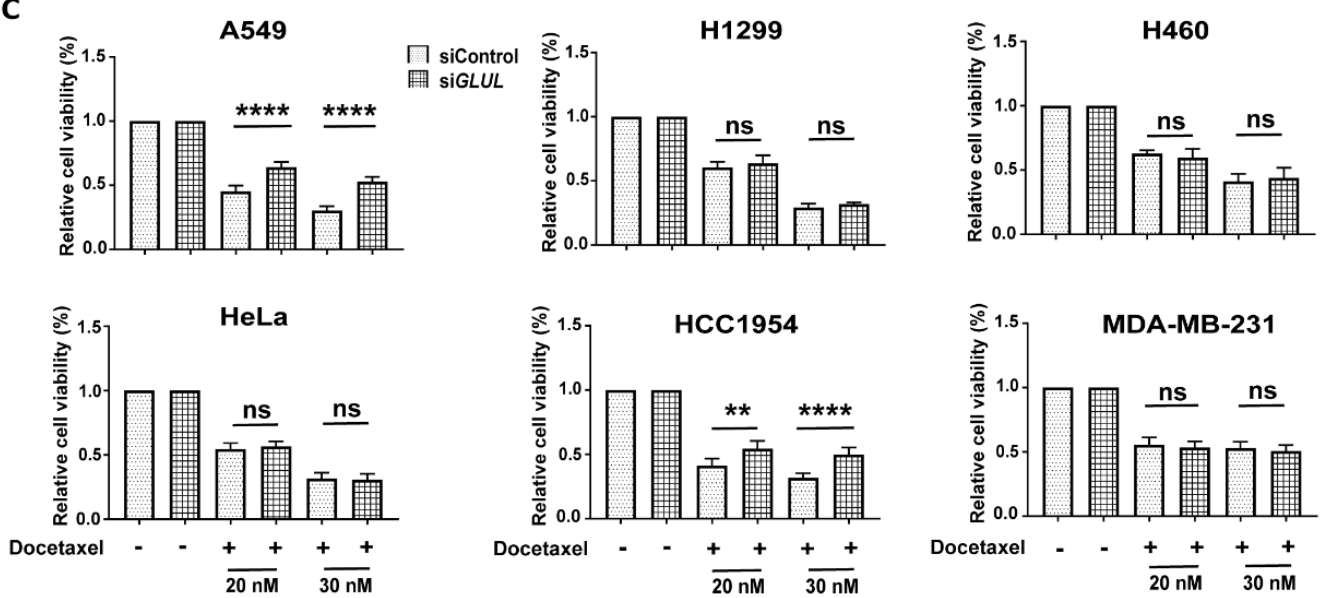

Figure 1. Reduced GLUL expression induced drug resistance. (A) GLUL (glutamate-ammonia ligase) protein expression was analyzed in different cancer cell lines. (B) Cell lines were either transfected with scrambled (siControl) or with siRNA GLUL as noted, and levels of GLUL protein expression were analyzed by western blotting. The western blot membranes were subsequently probed with an anti-tubulin antibody to assess equal loading. The presence of GLUL and tubulin protein is indicated on the right side of each blot. The approximate location of various molecular weight markers is indicated on the left side of each blot. kDa, kilo Dalton. (C) GLUL knockdown cell lines were treated with 20 and $30 \mathrm{nM}$ of docetaxel for $72 \mathrm{~h}$, and the cell viability was quantified by MTS assay. The standard error (SE) bars in cell viability graphs represent means of three independent experiments. The data are shown as mean \pm SEM; $p$-values were determined using a two-tailed unpaired t-test; ${ }^{* * *} p \leq 0.0001 ;{ }^{* *} p \leq 0.002$; ns-not significant. 
A549

H1299

A
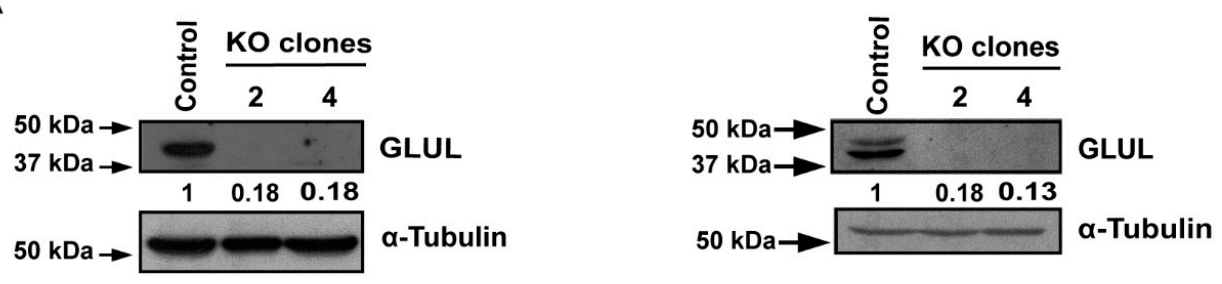

B
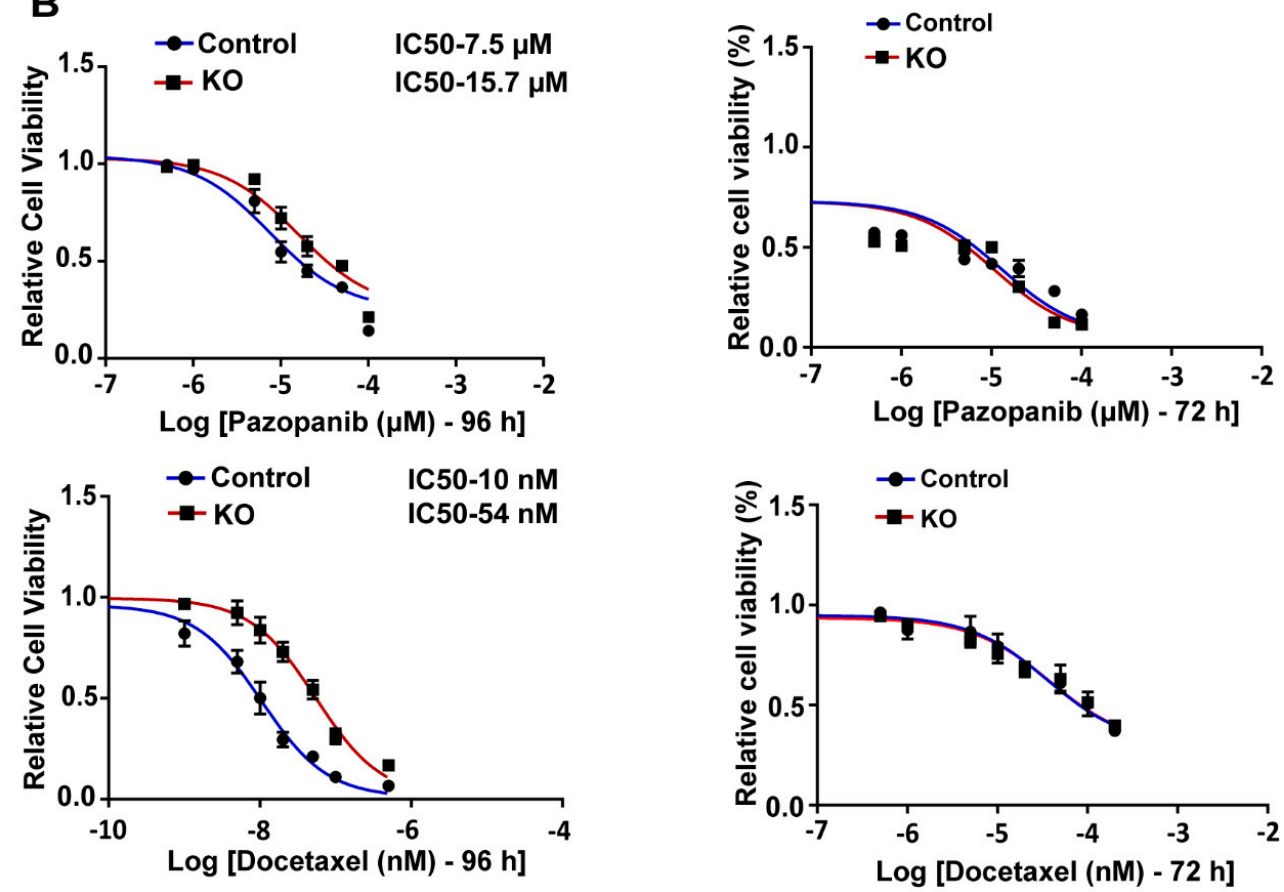

C
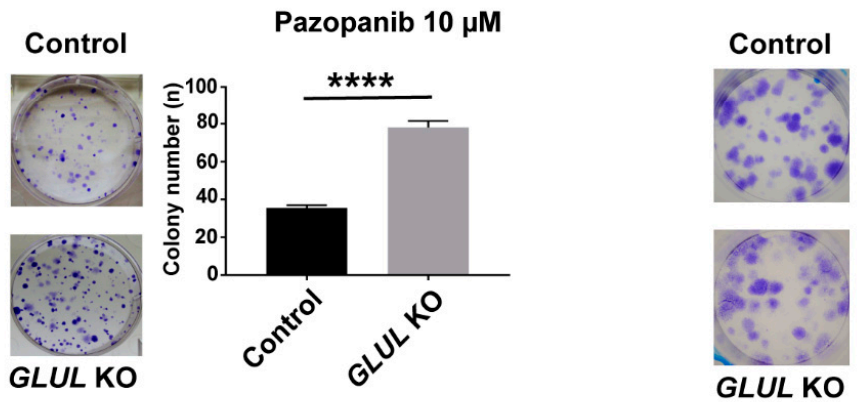

Pazopanib $10 \mu \mathrm{M}$
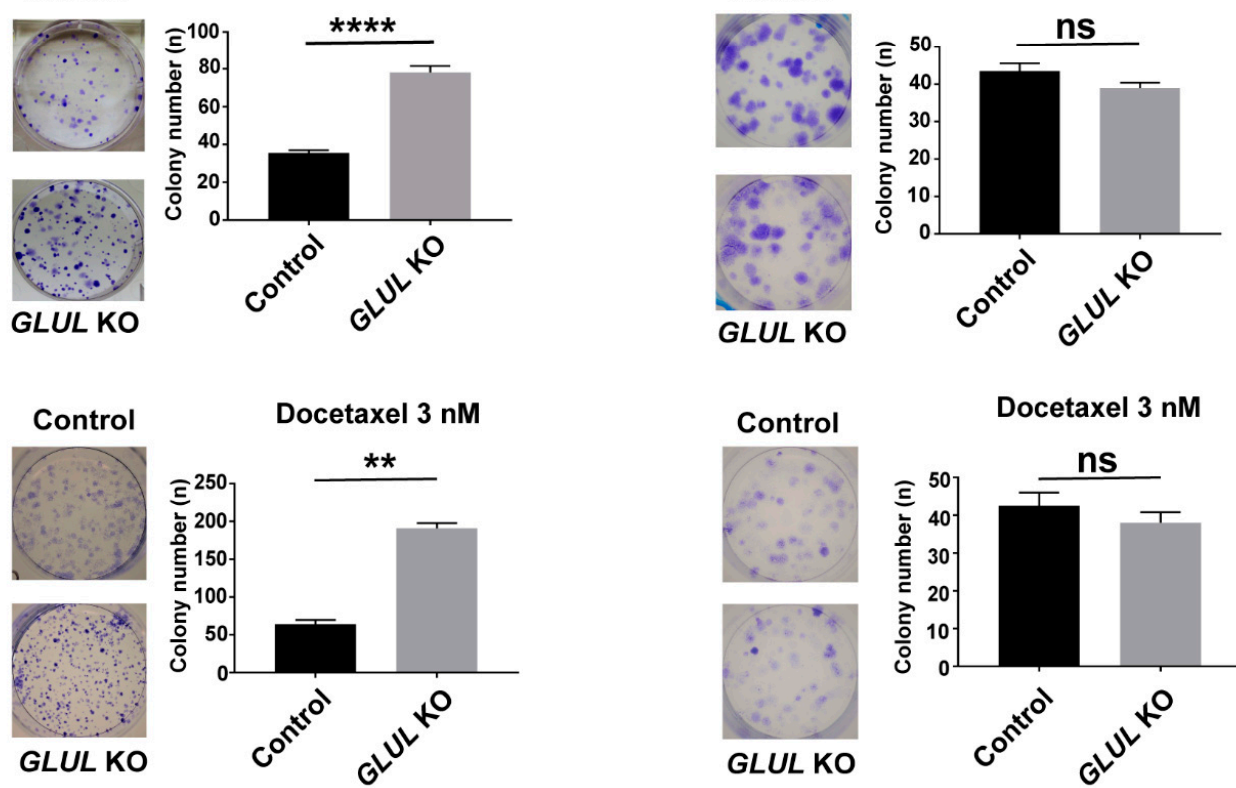

Docetaxel 3 nM

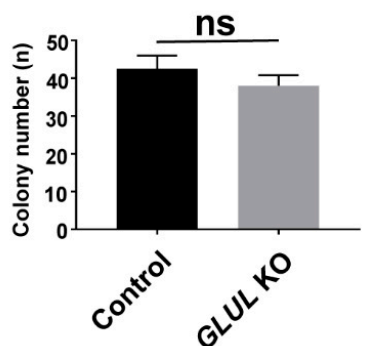

Figure 2. CRISPR/Cas9-mediated GLUL KO (knockout) conferred drug resistance in A549 but not in H1299 (A) A549 and H1299 cells were transfected with either negative controls (Lentiviral-empty 
vector) or Lentiviral CRISPR/Cas9-GLUL guide RNAs. Subsequently, cell lysates were analyzed by western blot (WB, see Methods) for GLUL expression, and individual clones devoid of GLUL expression were selected. The western blot membranes were subsequently probed with an anti-tubulin antibody to assess equal loading. The presence of GLUL and tubulin proteins are indicated on the right side of each blot. The signals for the GLUL and $\alpha$-tubulin proteins were quantified by densitometry, and the numbers below GLUL blots indicate levels of GLUL protein in each lane following normalization of the signals with tubulin levels. For comparison, the signal intensity of GLUL in the control lane was assigned an arbitrary value of 1 . The approximate location of various molecular weight markers is indicated on the left side of each blot. kDa, kilo Dalton. (B) A549 and H1299 control (DMSO-treated) or GLUL KO cells were treated with the indicated chemotherapeutic drugs, and the cell viability was analyzed by MTS assay after 72-96 h. IC 50 for each drug was determined as indicated. (C) A549 and H1299 control and GLUL KO cells were treated with the indicated drugs and subjected to clonogenic assay. Histograms represent a total number of colonies counted, and the representative images of crystal violet stained cells are shown. The standard error (SE) bars in cell viability assay and clonogenic assay represent the means of three independent experiments. The data are shown as mean $\pm \mathrm{SEM}$; $p$-values were determined using a two-tailed unpaired t-test; ${ }^{* * *} p \leq 0.0001 ;{ }^{* *} p \leq 0.004,{ }^{*} p \leq 0.01$, ns-not significant.

To confirm that GLUL loss of function potentiates drug resistance in A549 cells, we established stable cell lines expressing doxycycline-inducible shRNAs (shRNA 1, 2,3) that target GLUL (Supplementary Figure S1B). GLUL shRNA3 was significant in its ability to ablate GLUL protein expression upon doxycycline induction, and individual clones capable of expressing shRNA 3 were selected and expanded (Supplementary Figure S1C). We first examined the effect of various chemotherapeutic drugs, including dasatinib, pazopanib, and imatinib, on A549 cells expressing non-target or negative control shRNAs in comparison to A549 GLUL shRNA3 clone3 (cl3) (GLUL KD) cells through MTS assay. After $72 \mathrm{~h}$ of treatment, dose-response curves showed that 10-20 $\mu \mathrm{M}$ of each drug induced a significant loss of cell viability in A549 non-target or control cells, whereas in A549 GLUL KD cells, twice the dose of each of these drugs was required to achieve $50 \%$ of cell growth inhibition (Supplementary Figure S1D).

In order to differentiate the downstream effects of GLUL KO with respect to resistance, we also generated stable GLUL KO in non-resistant H1299 cells using the CRISPR-Cas9 method. Loss of GLUL protein expression was significant in $\mathrm{H} 1299$ cells, and lines expanded from individual clones were analyzed (Figure 2A). Additionally, we examined the cell viability in the presence of various pharmacological drugs in both H1299 control and H1299 GLUL KO Clone 2 (cl2) cells and observed no significant difference with their growth inhibition after $72 \mathrm{~h}$ (Figure 2B, Supplementary Figure S2D). Also, we performed clonogenic assays. Cells were grown in the presence of pazopanib $(10 \mu \mathrm{M})$, docetaxel $(5 \mathrm{nM})$, and dasatinib $(3 \mu \mathrm{M})$ for 12 days. There was no significant difference in colony formation between control and GLUL KO cells (Figure 2C, Supplementary Figure S2E). This suggested that the ability of GLUL KO to confer drug resistance was confined to specific cancer cell types.

\subsection{GLUL KO-Associated Drug Resistance Correlates with Apoptosis}

We next investigated the cellular pathway of chemotherapeutic drug resistance associated with GLUL KO in A549 cells in relation to apoptosis. Cleavage of poly(ADP-ribose) polymerase 1 (PARP-1) is a prominent marker of early apoptosis $[27,28]$. Western blot analysis showed that 10 and $20 \mu \mathrm{M}$ of pazopanib and imatinib; 20 and $30 \mathrm{nM}$ of docetaxel, as well as 5 and $10 \mu \mathrm{M}$ of dasatinib, stimulated PARP cleavage in A549 control cells. In contrast, GLUL KO cells displayed no, or reduced, PARP cleavage after $12 \mathrm{~h}$ treatment (Figure 3). Consistently, we observed the same effect following GLUL KD in A549 shRNA3 cl3 cells (Supplementary Figure S3). Our results showed that GLUL loss of function mediated a poor response to chemotherapeutic drugs by downregulating the apoptotic pathway in A549 cells. 
A549
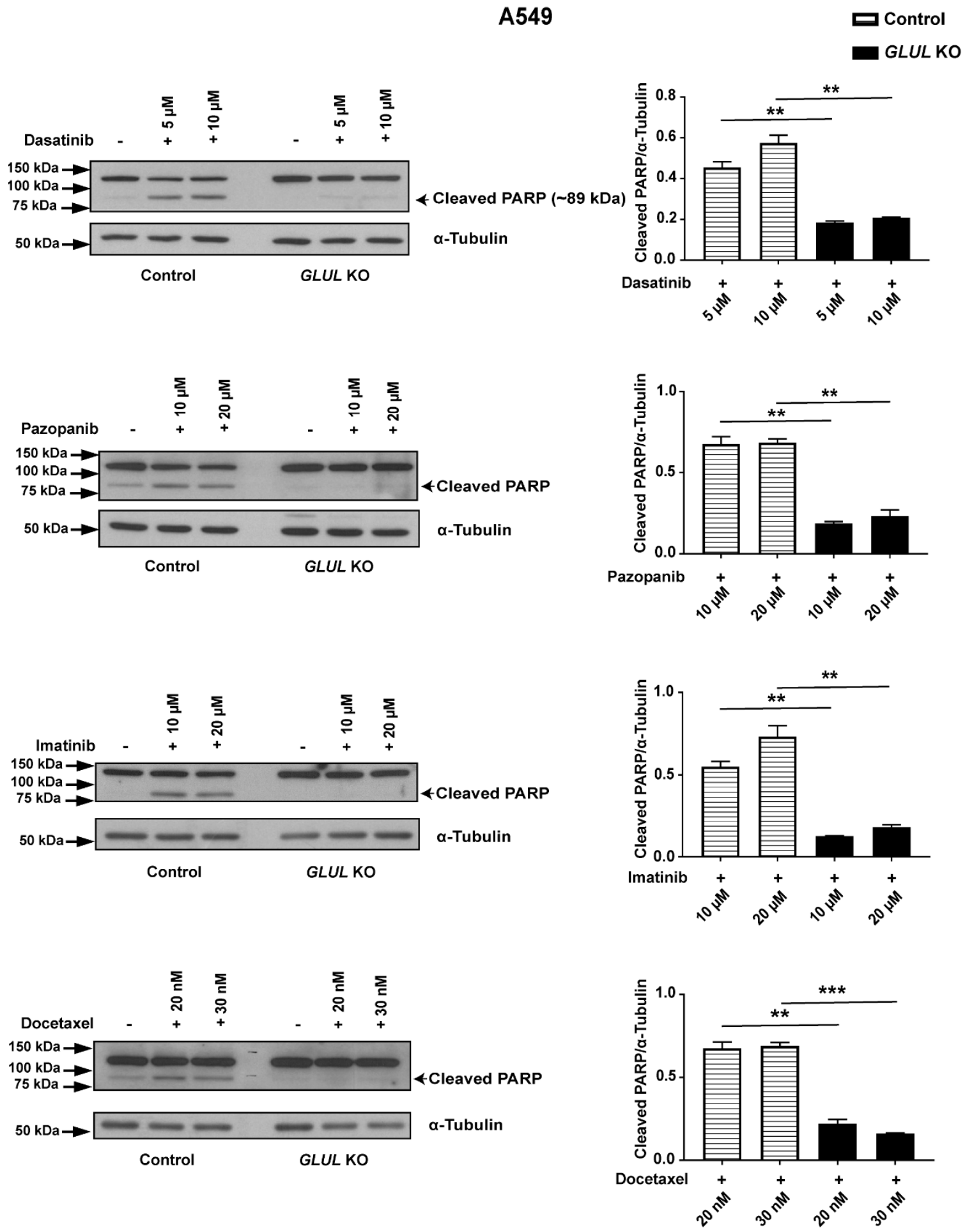

Figure 3. GLUL KO drug-resistant cells displayed reduced apoptosis. A549 control (DMSO-treated) or GLUL KO ( $\mathrm{cl} 3)$ cells were treated with various chemotherapeutic drugs for 12-18 h, as indicated. Subsequently, the expression of poly(ADP-ribose) polymerase (PARP)/cleaved PARP and $\alpha$-tubulin were analyzed by western blot. Quantification of protein levels is presented on the ride side of each western blot, and the approximate location of various molecular weight markers is indicated on the left side of each blot. $\mathrm{kDa}$, kilo Dalton. The data are shown as mean \pm SEM; $p$-values were determined using a two-tailed unpaired t-test; ${ }^{* *} p \leq 0.001$. 


\subsection{GLUL Knockout Cells Display Glucose Dependence}

Previously, we have shown that drug-resistant ALL cells with reduced GLUL transcription were characterized by reduced glutamine and increased glucose dependency [14]. Therefore, we explored the effects of reducing the concentrations of glutamine and glucose in the media on cell proliferation using MTS assay. No relative difference was observed between GLUL KO A549 and H1299 cells in proliferation under reduced media glutamine levels (Supplementary Figure S4). However, reducing the glucose concentration impacted significantly the proliferation of drug-resistant GLUL KO A549 cells compared to drug-sensitive A549 control cells (Figure 4). To understand this phenomenon, we treated both A549 and H1299 (control/ko) cells with 5 mM of 2-deoxy-D-glucose (2-DG), a glycolysis inhibitor [29] under various concentrations of glucose, and the cell viability was assessed through MTS assay. Indeed, A549 KO cells displayed higher sensitivity to 2-DG treatment with respect to their controls. This effect was not observed for H1299 cells (Figure 4). Thus, the drug resistance phenotype appeared to be connected with an increased requirement for glucose or increased dependence on glycolytic function.

\subsection{A Metabolic Phenotype Involving the Malate-Aspartate Shuttle Supports Drug Resistance Associated with GLUL KO}

To separate the resistance phenotype from the effects of GLUL KO, we compared two GLUL KO lines A549 (resistant) with H1299 (non-resistant). We performed targeted relative-quantitative profiling of 80 different metabolites in both A549 and H1299 cells. Several metabolites were uniquely associated with significant differences in only one of the cell line pairs when comparing $\mathrm{KO}$ to the negative control (Supplementary Figure S5A,B). Interestingly, glutamine accumulated when GLUL was knocked down, regardless of the drug resistance phenotype (Supplementary Figure S5A,B). It would be expected that a reduced capacity for de novo glutamine synthesis in the GLUL KO cell lines would affect glutamine uptake and/or the rate of glutaminolysis (conversion of glutamine to glutamate and $\alpha$-ketoglutarate). Indeed, the glutamine transporter SLC1A5 (Solute Carrier Family 1 Member 5) and the glutamate synthesizing enzyme, GLS, both showed increased expression in GLUL KD conditions (Supplementary Figure S6).

This prompted us to probe glutamine uptake and glutaminolysis flux by feeding cells with ${ }^{13} \mathrm{C}_{5}$ labeled glutamine. Quantifying the \%-fractional labeling after $24 \mathrm{~h}$ revealed how much of the intracellular pool of a metabolite was labeled with a stable isotope through uptake or biosynthesis (Supplementary Figure S5C). A small quantitative difference in glutamine levels between $\mathrm{KO}$ and control cells was observed (Supplementary Figure S5C). For glutamate, no difference between the cell lines was found, indicating that glutamine uptake was slightly increased when GLUL was reduced. However, the conversion of glutamine to glutamate was not altered following GLUL KO. The reduced $\%$-fractional labeling of $\alpha$-ketoglutarate was observed in both $\mathrm{KO}$ cell lines, suggesting that a reduced rate of glutaminolysis was mediated through reduced GLUL activity. As this was observed in both resistant and non-resistant cells, it could be concluded that altered glutaminolysis flux does not support resistance. When cells were exposed to either ${ }^{13} \mathrm{C}_{5}$ glutamate or ${ }^{13} \mathrm{C}_{5}$ glucose, a similar reduction in the labeling of $\alpha$-ketoglutarate was observed (Supplementary Figure S7). Interestingly, de novo glutamine synthesis was barely detectable in any of the cell lines as the fraction of labeled glutamine in the presence of ${ }^{13} \mathrm{C}_{5}$ glutamate only represented about $1 \%$. This low level was reduced still further in KO cells (Supplementary Figure S7), suggesting that the direct catalytic activity of GLUL was not essential for sustaining intracellular glutamine levels in these cells. 

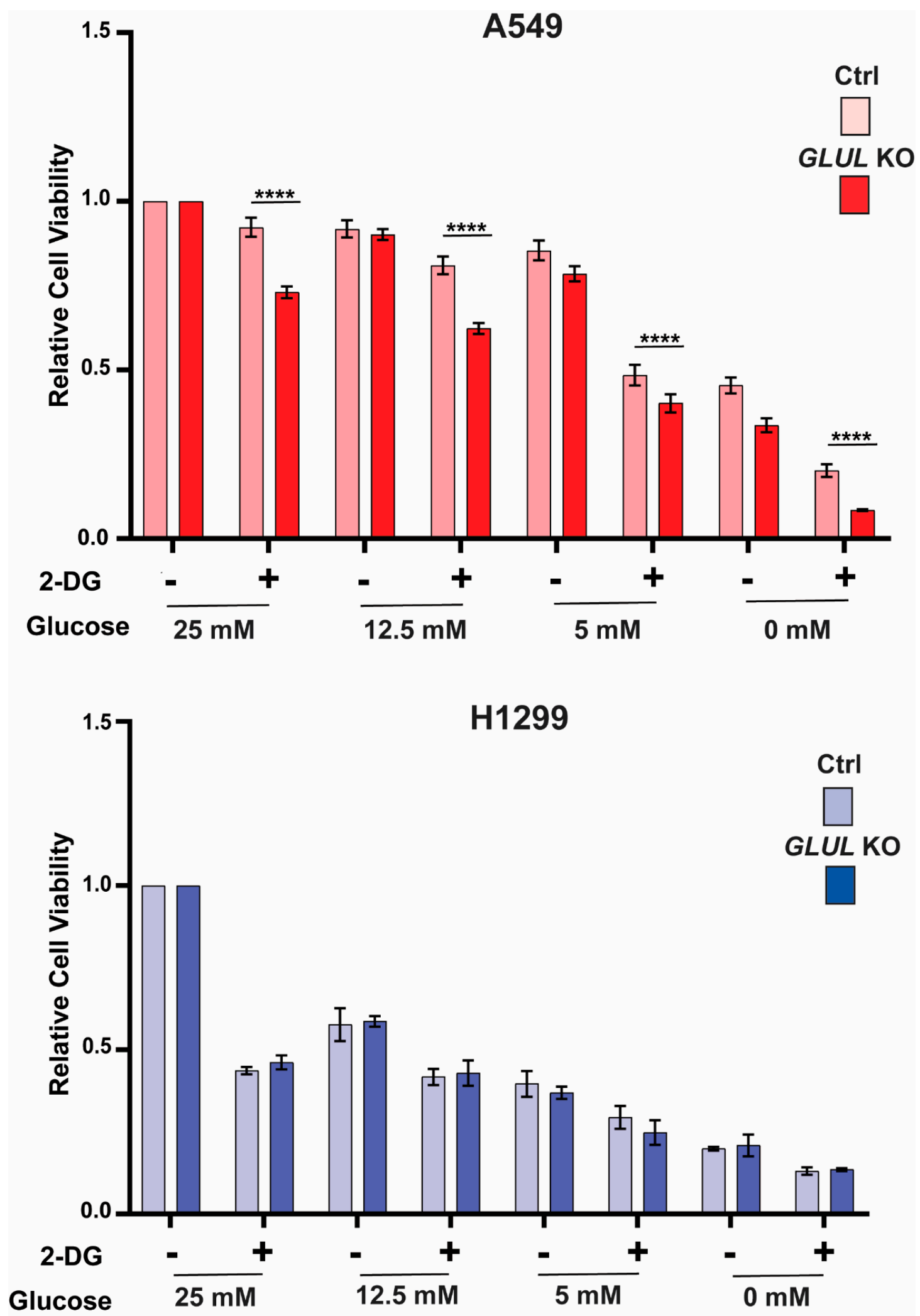

Figure 4. A549 GLUL KO cells displayed sensitivity to glucose deprivation. A549 and H1299 (control and GLUL KO) cells were cultured in various concentrations of glucose as indicated and were treated with $5 \mathrm{mM}$ of 2-DG for $72 \mathrm{~h}$, and the cell viability was analyzed by MTS assay. Data for each pair of cell lines (control/GLUL KO) was normalized to the data for the highest concentration of glucose (25 $\mathrm{mM}$ ), as shown above. The data are shown as \pm standard deviation. The standard deviation (SD) bars in the cell viability graph represent the means of three independent experiments. The data are shown as mean $\pm \mathrm{SD} ; p$-values were determined using a two-way ANOVA; ${ }^{* * * *} p \leq 0.001$. 
To investigate the fate of apparently increased glucose utilization in resistant GLUL KO A549 cells (Figure 4), we followed the \%-fractional labeling of glycolytic and TCA intermediates using ${ }^{13} \mathrm{C}_{6}$-labeled glucose and LC-MS. We were specifically interested in finding labeling patterns that differed between the resistant A549 GLUL KO cells and the non-resistant H1299 GLUL KO cells. Labeling of glycolytic intermediates showed similarly trending patterns for both cell lines as well as the TCA intermediates $\alpha$-ketoglutarate and citrate (Figure 5). However, malate and aspartate displayed a different pattern, with increased fractional labeling in A549 GLUL KO cells and reduced labeling in H1299 GLUL KO cells (Figure 5). An altered labeling pattern in malate and aspartate could imply that the malate-aspartate shuttle was linked to the drug-resistant phenotype. Increased flux through the malate aspartate shuttle could work together with increased glucose utilization towards increasing metabolic fitness through increased capacity for NADH production.

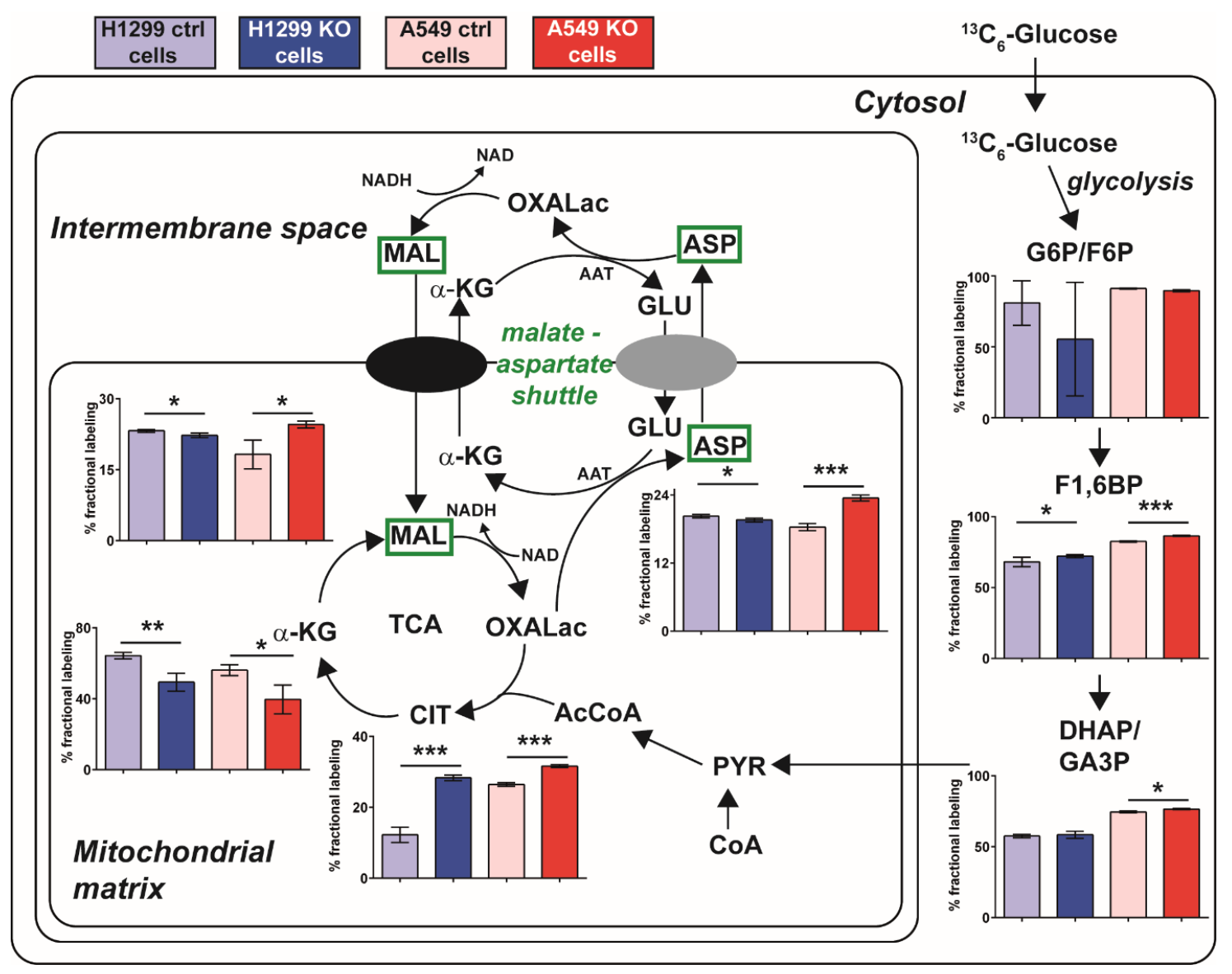

Figure 5. Increased flux through the malate-aspartate shuttle mediated drug resistance in A549 GLUL KO cells. Schematic illustration of a cell with mitochondria (intermembrane space + mitochondrial matrix). Superimposed are \%-fractional labeling for various metabolic intermediates; glucose-6-phosphate/fructose-6-phosphate (G6P/F6P), fructose-1,6-bisphosphate (F1,6BP), dihydroxyacetone phosphate/glyceraldehyde-3-phosphate (DHAP/GA3P), citrate (CIT), $\alpha$-ketoglutarate $(\alpha-K G)$, malate (MAL), aspartate (ASP), glutamate (GLU), oxaloacetate (OXALac), acetyl-coenzyme-A (AcCoA), pyruvate (PYR), coenzyme-A (CoA). Aspartate aminotransferase (AAT). Significance using unpaired students t-test ${ }^{*}(<0.05),{ }^{* *}(<0.005),{ }^{* * *}(<0.0005)$.

\subsection{Inhibition of the Malate-Aspartate Shuttle Reveals Metabolic Vulnerability Associated with Resistance}

The malate-aspartate shuttle is associated with increased metabolic fitness in a number of cancer cell types [26] and has been implicated in cancer cell proliferation [30,31]. Therefore, we hypothesized that GLUL ablation could mediate an increased capacity of the malate-aspartate shuttle. Thus, even in 
the absence of chemotherapeutic drugs, GLUL KO cancer cells should be more dependent on the support of this shuttle for mitochondrial NADH production and more sensitive to its inhibition. We tested this hypothesis by application of aminooxyacetic acid to inhibit the aspartate aminotransferase (AAT) (Figure 5), an enzyme that supports the malate-aspartate shuttle [32]. Aminooxyacetic acid had an $\mathrm{IC}_{50}$ of $11.5 \mu \mathrm{M}$ in resistant GLUL KO A549 cells (Figure 6) and an $\mathrm{IC}_{50}$ of $28 \mu \mathrm{M}$ in non-resistant control A549 cells. No such effects were observed in non-resistant H1299 GLUL KO cells (Supplementary Figure S8). These results provided evidence for a mechanism of drug resistance in GLUL ablated cells that were mechanistically supported through increased dependence on glycolysis linked with higher dependence on the malate-aspartate shuttle.

A549

A

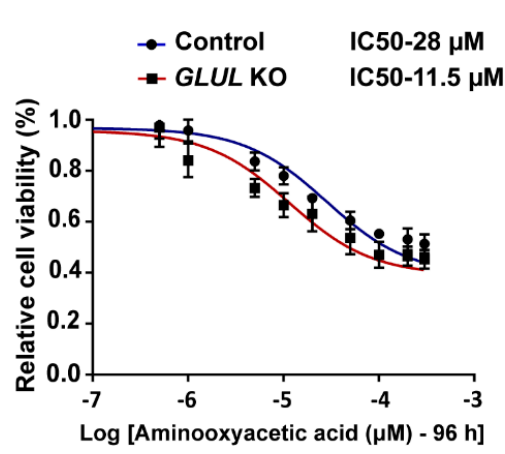

B
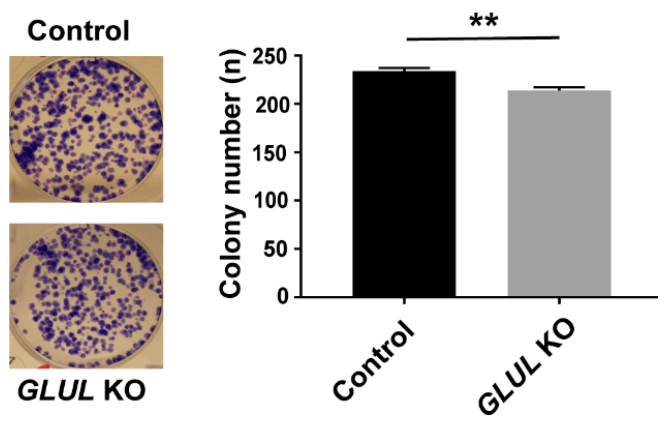

C

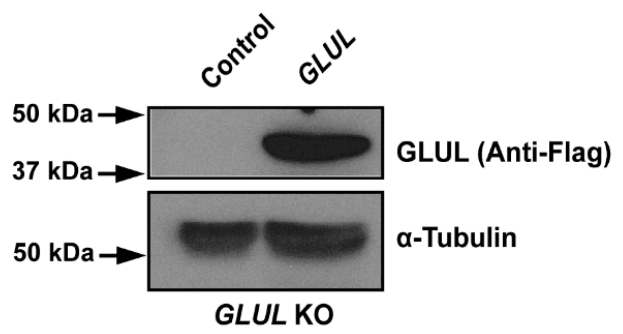

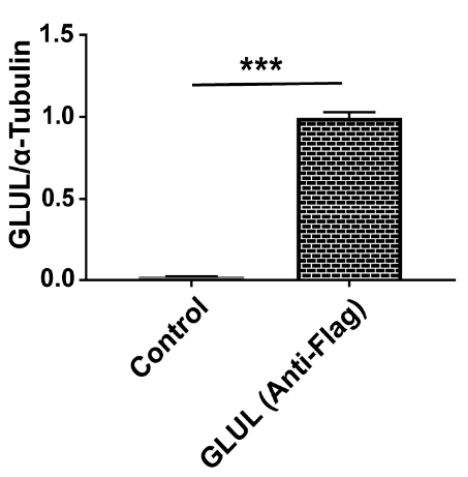

D

KO-Control-Docetaxel

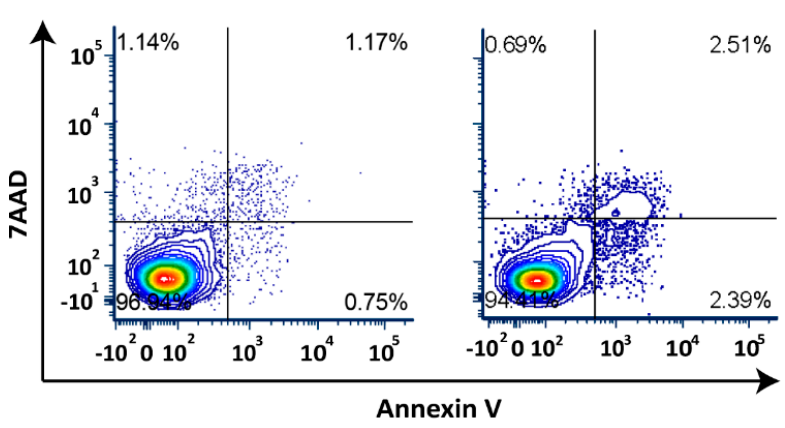

KO-GLUL-Docetaxel

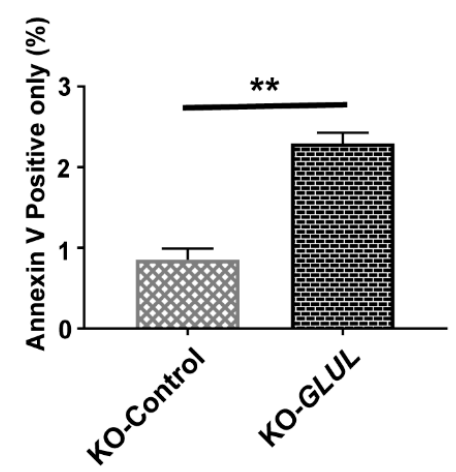

Figure 6. Targeting the malate-aspartate shuttle or GLUL expression re-sensitized drug-resistant A549 GLUL KO cells. (A) A549 GLUL KO/control cells (DMSO-treated) or treated with inhibitor aminooxyacetic acid at the concentrations were indicated. The cell viability was analyzed by MTS assay, and the $\mathrm{IC}_{50}$ values were determined as noted above (negative control $(28 \mu \mathrm{M})-95 \%$ confidence interval (CI)-2.245e-005 to 3.591e-005 and GLUL KO (11.5 $\mu \mathrm{M})-95 \%$ CI-8.416e-006 to 1.581e-005). The standard error (SE) bars in the cell viability curve represent means of three independent experiments. 
(B) Both control and GLUL KO cells were treated with the mentioned dose of aminooxyacetic acid for 12 days and subjected to colony formation assay. The column in the histogram represents the total number of colonies counted, and the colonies were photographed. (C) For GLUL overexpression, Flag-pcDNA3.1-GLUL cDNA clone and Flag-pcDNA3.1-empty vector were transfected in KO cells, and after $72 \mathrm{~h}$, the cell lysates were analyzed by western blotting (WB) as in methods for GLUL expression levels, as shown above. The western blot membranes were subsequently probed with an anti-tubulin antibody to assess equal loading. The presence of Flag-tag-GLUL protein is indicated on the right side of the blot, and the quantification of protein levels are represented. The approximate location of various molecular weight is indicated on the left side of each blot. kDa, kilodalton. (D) Flow cytometric analysis of apoptosis assay; A549 GLUL KO cells expressing Flag-pcDNA3.1-empty vector were treated with DMSO (control), and Flag-pcDNA3.1-GLUL were treated with docetaxel (20 nM) treatment for 12 $\mathrm{h}$, and the cells were stained with 7-Aminoactinomycin D (7-AAD) and annexin V. The \% of annexin V positive cells were measured and represented. The standard error (SE) bars, in the apoptosis assay, represent the means of two independent experiments. The data are shown as mean \pm SEM; $p$-values were determined using a two-tailed unpaired t-test; ${ }^{* *} p \leq 0.01,{ }^{* * *} p \leq 0.001$.

\subsection{Rescuing GLUL Expression Sensitizes A549 KO Cells to Drugs}

To verify that GLUL KO is responsible for the drug-resistant phenotype, we verified that GLUL sensitization to drug treatment could be rescued by GLUL re-expression in GLUL KO A549 cells. A Flag-tagged GLUL expression cassette was introduced in KO cells, and GLUL expression levels were analyzed by western blotting. Subsequently, A549 KO expressing Flag-GLUL and A549 KO cells were treated with docetaxel $(20 \mathrm{nM})$ for $12 \mathrm{~h}$ and were analyzed for apoptosis by annexin $\mathrm{V}$ staining. Flag-GLUL re-sensitized A549-KO cells to docetaxel (Figure 6), confirming that GLUL expression influenced drug responsiveness in a specific cancer cell type.

\section{Discussion}

With the advancement in our understanding of cancer metabolism, new opportunities for more efficient and less toxic cancer therapies will develop by exploiting the metabolic vulnerabilities of cancer cells that are not present in normal proliferating cells [33]. Targeting the mechanisms of drug resistance is a focal point in this endeavor, as these mechanisms will enable certain cancer cells to survive initial therapy and ultimately cause disease relapse. Recent findings from our lab have suggested that drug-resistant ALL cells lack GLUL transcription [14]. Importantly, we showed here that GLUL ablation conferred drug resistance in A549 NSCLC cells, and that this resistance was supported mechanistically through modulation of glycolytic flux linked with an upregulation of malate-aspartate transport in mitochondria. This shuttle is associated with increased metabolic fitness by enhancing the capacity of the cellular respiration $[25,26]$. Finally, targeting AAT as an essential component of the malate-aspartate shuttle [32,34] with aminooxyacetic acid had a larger impact on the viability of drug-resistant A549 GLUL KO cells and their drug-sensitive control counterparts. These findings supported a mechanistic model where GLUL ablation increased both glycolytic flux and the malate-aspartate shuttle concurrently. This increased the metabolic fitness of cells, aiding cell survival under drug selective pressure.

Recent studies in vivo have shown that the role of glutamine metabolism in cancer may be more complicated than previously understood [35]. The type of oncogene associated with a tumor, as well as the localization of the tumor tissue, appears to dictate the level of glutamine anaplerosis/cataplerosis in relation to the TCA cycle [36]. Furthermore, a mouse NSCLC model did not catabolize glutamine through glutaminolysis at all but depended solely on glucose-derived carbon flux [37]. Several studies have revealed a prognostic value of GLUL expression in different cancer types [21-24]. Further, the ablation of GLUL in SK-BR-3 cells reduced their proliferation [22]. Here, we showed that knockdown of GLUL induced drug resistance in A549 (NSCLC) and HCC1954 (breast cancer) cells (Figure 1A-C). Consistently, GLUL KD and/ or KO in A549 cells displayed drug resistance in a cell viability assay. 
Additionally, both GLUL KD and KO also displayed reduced or no PARP cleavage in response to a range of anticancer drugs (Figure 3, Supplementary Figure S3), supporting the conclusion that GLUL ablation suppressed apoptosis under selective pressure. Thus, mutations leading to reduced GLUL transcription should provide a survival advantage for cancer cells. To support this prediction, we queried the public database resource Kaplan-Meier plotter [38], which combines mRNA expression with patient survival data. We found strong significance for a correlation between reduced survival and lower GLUL mRNA expression in both breast and lung cancer patient cohorts (Supplementary Figure S9).

The multidrug-resistant protein (MDR1, ABCB1 or P-glycoprotein) has been associated with drug failure in patients $[8,9]$. The expression of this protein was not regulated by GLUL ablation in our study (Figure S6). Nor could we correlate the rate of cell growth to drug resistance, consistent with our western blot analysis of proliferating cell nuclear antigen (PCNA), a well-known cellular marker for proliferation [39] (Supplementary Figure S6). These findings suggest that the resistance is not driven by an increased expression of drug efflux pump or by reduced proliferation.

Previously, we observed altered dependence on exogenous glucose and glutamine for proliferation in resistant ALL cells lacking GLUL [14]. In the present study, we observed an increased dependence on glucose for proliferation in drug-resistant A549 GLUL KO (Figure 4), suggesting that cellular energetics were involved in driving the resistance phenotype. Interestingly GLUL KO cells did not show glutamine dependence that could be associated with a resistance phenotype (Supplementary Figure S4). To pinpoint changes in metabolism that could underlie the observed drug resistance phenotype, we used targeted metabolic profiling screening in GLUL KO/control pairs of A549 and H1299 cells (Supplementary Figure S5A,B). Metabolic profiling data revealed that both A549 and H1299 GLUL KO cells accumulated glutamine. This observation was supported mechanistically by increased SLC1A5 expression upon doxycycline-induced GLUL KD (Supplementary Figure S6). However, stable isotope labeling did not result in measurable increased ${ }^{13} \mathrm{C}_{5}$-glutamine uptake over $24 \mathrm{~h}$ (Supplementary Figure S5C). Additionally, flux from ${ }^{13} \mathrm{C}_{5}$-glutamine to ${ }^{13} \mathrm{C}_{5}$-glutamate (glutaminolysis) was analyzed by calculation of the \%-fractional labeling of ${ }^{13} \mathrm{C}$ in glutamate (Supplementary Figure S7). There was no increase in labeled glutamate in A549 or H1299 GLUL KO cells, suggesting that altered glutaminolysis did not directly drive the observed drug resistance mechanism. This was further supported by measuring a proportionally similar decrease in \%-fractional labeling in $\alpha$-ketoglutarate in both resistant and sensitive A549 and H1299 GLUL KO cells (Supplementary Figure S5C).

With the apparently increased requirement of glucose for proliferation (Figure 4) in A549 GLUL $\mathrm{KO}$ cells, we probed labeling through glycolysis and the TCA cycle using ${ }^{13} \mathrm{C}_{6}$-glucose to gauge if there was a discrepancy in the \%-fractional labeling intermediate metabolites between A549 and H1299 GLUL KO cells. Distinctively, the TCA intermediate malate displayed increased labeling in A549 GLUL KO cells and reduced labeling in H1299 GLUL KO cells compared to their respective controls. The same labeling patterns were also observed for aspartate (Figure 5). Malate and aspartate are coupled in a shuttle-facilitating transport of electrons into the mitochondrial matrix from the intermembrane space/cytosol (i.e., the malate-aspartate shuttle), enhancing cellular respiration $[40,41]$. This shuttle provides increased metabolic fitness in various cells for proliferation [26,30,31]. In addition, the cataplerotic function of TCA cycle intermediates for a range of other metabolite classes, including those involved in lipid and nucleotide biosynthesis [12,42], suggesting a wider potential role for increased flux in malate and aspartate that may represent a critical point in metabolic rewiring, which mediates drug resistance in A549 GLUL KO cells.

Previous studies have indicated that pharmacological targeting of the malate-aspartate shuttle can inhibit cancer cell proliferation [30,31]. Administration of aminooxyacetic acid to target aspartate aminotransferase [34], which plays an integral part in the malate-aspartate shuttle (Figure 5), revealed that resistant A549 GLUL KO cells were significantly more sensitive to drug treatment compared to A549 control cells; illustrated with both viability and a colony-forming assay (Figure 6). Furthermore, a recent study reported that GLUL overexpression sensitized NSCLCs to anti-cancer drug gefitinib 
treatment [43]. Consistent with that finding, rescuing expression of GLUL in A549 KO cells re-sensitized cells to docetaxel treatment with respect to their controls (Figure 6), suggesting that GLUL might play a vital role in determining drug sensitivity in targeted therapies.

\section{Material and Methods}

\subsection{Cell Culture and Reagents}

NSCLCs A549 (ATCC ${ }^{\circledR}$ CCL-185 ${ }^{\mathrm{TM}}$, Manassas, VA, USA), H1299 (ATCC ${ }^{\circledR}$ CRL-5803 ${ }^{\mathrm{TM}}$ ), H460 (ATCC ${ }^{\circledR}$ HTB-117 ${ }^{\mathrm{TM}}$ ), the cervical cancer cell line HeLa (ATCC ${ }^{\circledR}$ CCL-2 ${ }^{\mathrm{TM}}$ ), and breast cancer cell lines HCC1954 (ATCC ${ }^{\circledR}$ CRL-2338 ${ }^{\mathrm{TM}}$ ) and MDA-MB-231 (ATCC ${ }^{\circledR}$ HTB-26 $^{\mathrm{TM}}$ ) were acquired from the American Type Culture Collection and maintained according to the ATCC's recommendations. DMEM (41965039), MEM (10370047), RPMI-1640 (72400021), and FBS (16000044) were obtained from Thermofisher Scientific (Thermofisher Scientific, Waltham, MA, USA). Cell culture media were supplemented with $10 \%(v / v)$ fetal bovine serum (FBS), 100 units $/ \mathrm{mL}$ of penicillin, and $100 \mu \mathrm{g} / \mathrm{mL}$ of streptomycin. Cells were cultivated in a humidified atmosphere at $37^{\circ} \mathrm{C}$ and $5 \%(v / v) \mathrm{CO}_{2}$.

FDA approved chemotherapeutic drugs: dasatinib (approved for chronic myeloid leukemia and ALL), pazopanib (multi-targeted tyrosine kinase inhibitor, approved for renal cell carcinoma (RCC) [44] and soft tissue sarcoma [45]), docetaxel (inhibitor of microtubule formation [46], approved for breast cancer, head and neck cancer, stomach cancer, prostate cancer, and NSCLC), and cisplatin (induces apoptosis via DNA damage [47], approved for testicular cancer, ovarian cancer, cervical cancer, breast cancer, bladder cancer, head and neck cancer, esophageal cancer, lung cancer, mesothelioma, brain tumors, and neuroblastoma) were purchased from Selleckchem (Houston, TX, USA).

Aminooxyacetic acid (C13408) was obtained from Sigma Aldrich (D2650, Sigma Aldrich, St. Louis, MO, USA), dissolved in dimethyl sulfoxide (DMSO) at a stock concentration of $50 \mathrm{mM}$, and stored at $-80^{\circ} \mathrm{C}$. Anti-GLUL (ab64613), anti-GLS (ab93434), anti-PCNA (ab29), and anti- $\alpha$-tubulin (ab4074) antibodies were purchased from Abcam (Cambridge, UK). Anti-GLUL (LS-C114787) was purchased from LifeSpan Biosciences Inc. (Seattle, WA, USA). Anti-cleaved PARP (5625), anti-MDR1 (12683), anti-AKT (4685), and anti-pAKT (4060) were from Cell Signaling Technology (Beverly, MA, USA). Secondary antibodies: HRP-conjugated anti-mouse (1721011), anti-rabbit (1721019) were from Bio-Rad (Hercules, CA, USA). Amersham ECL western blotting detection reagent (RPN2106) was purchased from GE Healthcare Lifesciences (Pittsburgh, PA, USA).

\subsection{GLUL siRNA Transfection}

Cells were seeded in 6-well plates $\left(2 \times 10^{5}\right.$ per well) for $24 \mathrm{~h}$ before transfection. After $12-18 \mathrm{~h}$, cells were transfected with either scrambled RNA $(20 \mathrm{nM})-(\mathrm{ON}-\mathrm{TARGETplus} \mathrm{Control} \mathrm{pool-Non-Targeting}$ pool-D-001810-10-05-Dharmacon, Lafayette, CO, USA) or GLUL siRNA (20 nM)-(ON-TARGET plus SMARTpool-Human GLUL-L-008228-01-0010_Dharmacon) for $72 \mathrm{~h}$ before treatment.

\subsection{Establishment of Inducible GLUL KD Cell Lines by shRNA Expression}

To knockdown GLUL expression, three individual SMARTvector Inducible Human GLUL shRNAs were designed as follows; shRNA1-V3sH7669-225458080 (GTCCGGATAGCTACGCCTA), shRNA2-V3SH7669-226004989 (GTTACAATCGGGACAACTG) targeting the 3'UTR, shRNA3V3SH7669-228686437 (TAGACGTCGGGCATTGTCC) targeting the ORF, and a Non-Target shRNA (VSC6584) as a negative control was purchased from Dharmacon. For lentiviral GLUL-shRNA transduction, the concentration of multiplicity of infection (MOI) 2.0 was optimized for each GLUL-shRNA expressing GFP, and the complete medium was added to HEK293T cells for $72 \mathrm{~h}$; then, the supernatant medium was collected, sterile filtered, and applied to A549 cells with $8 \mu \mathrm{g} / \mathrm{mL}$ polybrene and incubated for $72 \mathrm{~h}$. Subsequently, fresh media containing $10 \mu \mathrm{g} / \mathrm{mL}$ puromycin (ant-pr-1; Invivogen, San Diego, CA, USA) were replaced after $48 \mathrm{~h}$. From this pool of transduced cells, single cells expressing GFP were isolated for clonal expansion by FACS analysis. 
To induce GLUL knockdown, $2.0 \times 10^{5}$ cells passaged in media containing tetracycline-free FBS were seeded into each well of a 6-well plate, and $5 \mu \mathrm{g} / \mathrm{mL}$ doxycycline was added after $12 \mathrm{~h}$. Cells were analyzed after 48-96 h.

\subsection{Establishment of Stable GLUL KO Cell Lines by CRISPR/Cas9}

GLUL KO cells were generated using CRISPR/Cas9-mediated deletion. A transfer plasmid lentiCRISPRv2 with guide RNA1 (TGTTTCGGGACCCCTTCCGT)—Transcript: GLUL-201 ENST00000311223.9-falls in exon 4 (coding sequence/translation start site is in exon 3), and guide RNA2 (TATTACTGTGGTGTGGGAGC)—Transcript: GLUL-201 ENST00000311223.9-falls in exon 6 (coding sequence) were co-transfected into HEK293T cells with packaging plasmids pVSVg (8454; Addgene, Watertown, MA, USA) and psPAX2 (12260; Addgene) [48,49]. As a negative control, LentiCRISPR V2 negative control/human non-targeting guide RNA-(Forward primer: 5'-ACGGAGGCTAAGCGTCGCAA-3', Reverse primer: 5'-TTGCGACGCTTAGCCTCCGT-3'; from GeCKO v2 libraries) was co-transfected into HEK293T cells with packaging plasmids pVSVg and psPAX2 using Lipofectamine LTX reagent (Thermo Fisher Scientific, Waltham, MA, USA). After $48 \mathrm{~h}$, virus-containing supernatant was collected and filtered $(0.45 \mu \mathrm{M})$. This was added to cells together with $8 \mu \mathrm{g} / \mathrm{mL}$ polybrene and complete medium. After incubating for $48 \mathrm{~h}$, transduced cells were selected with $10 \mu \mathrm{g} / \mathrm{mL}$ puromycin. After 1 week of antibiotic selection, single cells were sorted by FACS analysis for clonal expansion.

\subsection{GLUL Re-Expression in A549 GLUL KO Cells}

GLUL KO cells were seeded a day before transfection in 6-well plates $\left(3 \times 10^{5}\right.$ per well). After $12 \mathrm{~h}$, cells were transfected with either GLUL-ORF cDNA clone-NM_001033044.3 (constructed with pcDNA3.1+/C-(K)-DYK vector) or empty vector (pcDNA3.1+/C-(K)-DYK), purchased from Genscript (Piscataway, NJ, USA). For transfection, $5 \mu \mathrm{g}$ empty vector or GLUL-ORF cDNA clone, as well as Lipofectamine 3000, were diluted with Opti-MEM. After 5 min of incubation, diluted DNA and Lipofectamine 3000 were combined and incubated for 15-20 min to allow DNA-Lipofectamine 3000 complexes to form. The complexes were added with complete medium to cells and incubated. After $72 \mathrm{~h}$ of transfection, cells were subjected to apoptosis assay and western blot analysis.

\subsection{MTS Assay and Western Blot Analysis}

Cell growth inhibition was analyzed by MTS Colorimetric Assay Kit (ab197010; Abcam). Cells were seeded in 96-well plate $\left(5 \times 10^{3}\right.$ per well) in six replicates and then treated with chemotherapeutic drugs, including dasatinib, imatinib, pazopanib, docetaxel (1 nM to $50 \mathrm{nM})$, cisplatin, 2-DG (50 mM), and aminooxyacetic acid at various concentrations $(500 \mathrm{nM}$ to $300 \mu \mathrm{M})$ for $48-96 \mathrm{~h}$. Negative controls were treated with $0.1 \%(v / v)$ DMSO in serum-free medium. After drug treatment, cells were treated with $20 \mu \mathrm{L}$ MTS reagent per well for 1-3 h, and then the absorbance was measured at $490 \mathrm{~nm}$ (Tecan Infinite 200 PRO, Männedorf, Switzerland). The background signal was normalized with the signal from adjacent wells containing medium and MTS reagent.

Western blot analyses were performed according to standard procedures. For protein extraction, cells were lysed with RIPA buffer (R0278; Sigma Aldrich) containing Protease Inhibitor Cocktail (P8340; Sigma Aldrich) and Phosphatase Inhibitor Cocktail (P2850; Sigma Aldrich). Extracted proteins were separated by SDS-PAGE using 10\% polyacrylamide gels and then transferred to a PVDF membrane (Millipore, Darmstadt, Germany). After blocking in 5\% milk or bovine serum albumin in Tris-buffer saline-Tween $20(0.1 \%)$ (TBS-T), blots were probed with primary antibodies and then secondary antibodies. Antibody binding was visualized using ECL reagent according to the manufacturer's instructions (GE Healthcare Life Sciences, Pittsburgh, PA, USA) following exposure to X-ray film (Agfa Healthcare NV, Mortsel, Belgium). The blots were re-probed with an antibody against $\alpha$-tubulin as a loading control. 


\subsection{Clonogenic Assay}

Cells were seeded into each well of a 6-well plate $\left(2 \times 10^{3}\right.$ per well), and after $12 \mathrm{~h}$, treated with drugs, including dasatinib (5 or $3 \mu \mathrm{M})$, pazopanib $(10 \mu \mathrm{M})$, imatinib $(10 \mu \mathrm{M})$, cisplatin $(5 \mu \mathrm{M})$, and docetaxel $(3 \mathrm{nM})$ in complete medium. Cells were grown for 12 days; then, cells were fixed with methanol/acetic acid (5:1) for $5 \mathrm{~min}$ and stained with crystal violet $0.5 \%$ solution (S25275B; Fisher Scientific, Hampton, NH, USA) for $30 \mathrm{~min}$. The total number of colonies per well were counted and compared to negative controls.

\subsection{Flow Cytometry (FACS)-Based Apoptosis Analysis}

Flow cytometry (FACS)-based assays were performed on A549 GLUL KO cells treated with docetaxel to analyze apoptosis. For apoptosis assay, PE-conjugated annexin V and 7-AAD staining was performed according to the manufacturer's protocol (BD Biosciences, San Jose, CA, USA). Data were acquired using BD LSR II flow cytometer, and the analysis was performed with FCS Express 6Plus software (De Novo Software, Pasadena, CA, USA).

\subsection{Metabolic Profiling and Stable Isotope Labeling and Tracing}

For targeted metabolomics, cells were seeded into a 6-well plate $\left(2 \times 10^{5}\right.$ cells/well $)$ and grown for $12 \mathrm{~h}$. Cells were then incubated with stable isotopes for $24 \mathrm{~h}$ and washed at least once with PBS, scraped, and transferred to a $1.5 \mathrm{~mL}$ Eppendorf tube. The cells were lysed, and metabolites were extracted using $200 \mu \mathrm{L}$ 90:10 methanol: $\mathrm{H}_{2} \mathrm{O}$ containing $0.36 \mu \mathrm{M}^{13} \mathrm{C}_{9}$-phenylalanine as an internal standard, to which acid-washed glass-beads $425-600 \mu \mathrm{M}$ from Sigma Aldrich (St. Louis, MO, USA) were added to constitute $\sim 50 \% v / v$ of the $90: 10 \mathrm{MeOH}: \mathrm{H}_{2} \mathrm{O}$ cell suspension and the cells were disrupted by shaking at $30 \mathrm{~Hz}$ for $2 \mathrm{~min}$ (Mixer Mill MM400, Retsch, Germany) using pre-chilled holding blocks ( $4^{\circ} \mathrm{C}$ ). Samples were centrifuged at $4{ }^{\circ} \mathrm{C} / 14,000 \mathrm{rpm}$ for $15 \mathrm{~s}$, after which $100 \mu \mathrm{L}$ of water was added, and samples were shaken again for $1 \mathrm{~min}$ for $15 \mathrm{~s}$ before the centrifugation step was repeated. Subsequently, $150 \mu \mathrm{L}$ of the supernatant was transferred to LC-MS glass vials, from which 2-10 $\mu \mathrm{L}$ was injected into an Agilent 1290 LC-system connected to either a 6550 or 6560 Agilent Q-TOF mass spectrometer (Agilent, Santa Clara, CA, USA). An electrospray ionization (ESI) source was used in all of the LC-MS measurements. Data were collected between $\mathrm{m} / \mathrm{z} 70$ to 1700 in positive/negative ion mode. The following ESI settings were used (Agilent Jetstream): gas temperature $300{ }^{\circ} \mathrm{C}$, gas flow $8 \mathrm{l} / \mathrm{min}$, nebulizer pressure 40 psi, sheet gas temperature $350^{\circ} \mathrm{C}$, sheet gas flow 11 , Vcap 4000 , fragmentor 100, Skimmer1 45, and OctapoleRFPeak 750. Metabolites were separated using reversed-phase chromatography (HSS T3 C18, $50 \times 2.1 \mathrm{~mm}$, $1.8 \mu \mathrm{M} 100 \AA$ (Waters, MA, USA)) or HILIC (iHILIC-Fusion (+), $100 \times 2.1 \mathrm{~mm}, 3.5 \mu \mathrm{M}, 100 \AA$, Hilicon $\mathrm{AB}$ (Umeå, Sweden)). For reversed-phase chromatography elution, solvents were $(A) \mathrm{H}_{2} \mathrm{O}, 0.1 \%$ formic acid and (B) 75:25 acetonitrile:isopropanol, 0.1\% formic acid. For separation, the following linear gradient was used (flow rate $0.5 \mathrm{~mL} / \mathrm{min}$ ), $\min 0: 0.1 \% \mathrm{~B}, \min 2: 10 \% \mathrm{~B}, \min 7: 99 \% \mathrm{~B}, \min 9: 99 \%$ $\mathrm{B}, \min 9.3: 0.1 \% \mathrm{~B}, \min 10.8: 0.1 \% \mathrm{~B}$. Column re-equilibration occurred from 9.8 to $10.7 \mathrm{~min}$ with an increase in flow rate to $0.8 \mathrm{~mL} / \mathrm{min}$. HILIC elution solvents were (A) $\mathrm{H}_{2} \mathrm{O}, 50 \mathrm{mM}$ ammonium formate (B) 90:10 (acetonitrile):( $\mathrm{H}_{2} \mathrm{O} 50 \mathrm{mM}$ ammonium formate), total concentration ammonium formate in $\mathrm{B}$ $5 \mathrm{mM}$. The chromatographic separation was achieved using the following linear gradient (flow rate $0.4 \mathrm{~mL} / \mathrm{min}), \min 0: 90 \% \mathrm{~B}$; $\min 4: 85 \% \mathrm{~B}, \min 5: 70 \% \mathrm{~B}, \min 7: 55 \% \mathrm{~B}, \min 10: 20 \% \mathrm{~B}, \min 10.01: 90 \%$ B, $\min 15: 90 \%$ B. Raw data were processed using Masshunter Profinder and the function batch target feature extraction (Agilent). Target feature libraries were obtained using synthetic standards in an in-house generated PCDL library (Agilent) through recording LC retention times and accurate masses. The internal standard ${ }^{13} \mathrm{C}_{9}$-phenylalanine was used for analytical quality monitoring and detected in all four separation/ionization modes. Ratios for $\mathrm{KO} / \mathrm{control}$ were calculated for all metabolite responses that were different $(p<0.05)$ between $\mathrm{KO}$ and control (ctrl) cells.

For stable isotope labeling, cells were seeded in a 6-well plate $\left(2.0 \times 10^{5}\right.$ cells/well) and grown overnight. After 12-18 h, complete DMEM containing 10\% FBS and stable isotopes, including 
${ }^{13} \mathrm{C}_{5}$-Glutamic acid (604860; Sigma Aldrich, St. Loius, MO, USA)-500 $\mu \mathrm{M},{ }^{13} \mathrm{C}_{5}$-Glutamine (605166; Sigma Aldrich)-500 $\mu \mathrm{M}$, and ${ }^{13} \mathrm{C}_{6}$-D-Glucose (CLM-1396-1; PR-22544; Lardon Fine Chemicals AB, Sweden)-12.5 mM, were added to cells with adjustment of the concentration of unlabeled glutamine or glucose in the culture medium to maintain $4 \mathrm{mM}$ and $25 \mathrm{mM}$, respectively. After $24 \mathrm{~h}$ of stable isotope incubation, cells were washed with PBS and subjected to metabolite extraction and LC-MS analysis, as described above. The \%-fractional labeling was calculated through data processing using the Batch Isotopologue Extraction function in Agilent Masshunter Profinder (B 08.00, Agilent).

\subsection{Statistical Analysis}

Statistical analysis was performed using GraphPad Prism software 7 (San Diego, CA, USA). All the mean values are the average value of all samples. The standard deviation (SD) is an indication of the variability of all samples. The sample mean is indicated by the standard error. The confidence level is expressed using a 95\% confidence interval (CI). All of the statistical tests were two-sided, and $p<0.05$ was considered to be statistically significant. For all in vitro experiments, at least three independent experiments were conducted. All quantification data presented in the study was an average of at least three independent experiments. All western blots represented are at least out of two independent experiments. All $\mathrm{IC}_{50}$ curves are nonlinear regression fit plotted against $\log$ (inhibitor) vs. response (three parameters) using Graphpad Prism software (San Diego).

\section{Conclusions}

Here, we showed that GLUL ablation induced resistance to several different cancer drugs in specific cell lines. This gain of function phenotype provided an advantage to cancer cells under therapeutic pressure. Drug-resistant cells depended more on exogenous glucose for proliferation. Stable isotope-labeled glucose provided evidence for increased flux through malate and aspartate in resistant cells. We interpreted this as an increased reliance on the malate-aspartate shuttle in resistant cells, and that the enhanced metabolic fitness that follows mechanistically supports the resistance phenotype and aids cells in their escape from therapeutic pressure.

Supplementary Materials: The following are available online at http://www.mdpi.com/2072-6694/11/12/1945/s1, Figure S1: GLUL KO/KD induces drug resistance. Figure S2: GLUL KO cells display drug resistance in A549 but not in H1299 cells. Figure S3: A549 GLUL KD exhibit reduced or no PARP cleavage. Figure S4. Reduced glutamine does not correlate with drug resistance in GLUL KO cells. Figure S5: Targeted metabolomics reveals glutamine accumulation in resistant cells without increased glutamine uptake or flux through the glutaminolysis pathway. Figure S6: Reduced GLUL expression induces glutaminolysis. Figure S7: \%-Fractional labeling of glutaminolysis intermediates. Glutamine (GLN), Glutamate (GLU), and alpha-ketoglutarate ( $\alpha-K G)$. Glutamate dehydrogenase (GLUD), Glutaminase (GLS), Glutamate-ammonia ligase (GLUL). Figure S8. H1299 KO cells do not display sensitivity to ATT inhibition. Figure S9: Reduced GLUL mRNA expression correlates with poor prognosis in breast and lung cancer patients.

Author Contributions: M.M. and A.N. conceived the project. R.K., A.N., M.M., J.D.G., A.S.S.K. and J.L.P. designed experiments. A.N., M.M., J.D.G., A.S.S.K. performed experiments. All authors analyzed results. M.M. and A.N. wrote initial draft of manuscript. All authors contributed towards subsequent versions of the manuscript.

Funding: The Kempe Foundations, grant number JCK-1621.

Acknowledgments: The Kempe Foundations, JCK-1621 is gratefully acknowledged for financial support (A.N). The authors wish to acknowledge The Swedish Metabolomics Centre (SMC) for making LC-MS instruments and technical support available.

Conflicts of Interest: The authors declare no conflict of interest.

\section{Abbreviations}

NSCLC: non-small cell lung cancer; GLUL, glutamate-ammonia ligase; KD, knockdown; KO, knockout. 


\section{References}

1. Stronach, E.A.; Alfraidi, A.; Rama, N.; Datler, C.; Studd, J.B.; Agarwal, R.; Guney, T.G.; Gourley, C.; Hennessy, B.T.; Mills, G.B.; et al. HDAC4-regulated STAT1 activation mediates platinum resistance in ovarian cancer. Cancer Res. 2011, 71, 4412-4422. [CrossRef]

2. Patel, C.; Stenke, L.; Varma, S.; Lindberg, M.L.; Bjorkholm, M.; Sjoberg, J.; Viktorsson, K.; Lewensohn, R.; Landgren, O.; Gottesman, M.M.; et al. Multidrug resistance in relapsed acute myeloid leukemia: EVIDENCE of biological heterogeneity. Cancer 2013, 119, 3076-3083. [CrossRef]

3. Haffner, M.C.; Mosbruger, T.; Esopi, D.M.; Fedor, H.; Heaphy, C.M.; Walker, D.A.; Adejola, N.; Gurel, M.; Hicks, J.; Meeker, A.K.; et al. Tracking the clonal origin of lethal prostate cancer. J. Clin. Investig. 2013, 123, 4918-4922. [CrossRef]

4. Leary, M.; Heerboth, S.; Lapinska, K.; Sarkar, S. Sensitization of Drug Resistant Cancer Cells: A Matter of Combination Therapy. Cancers 2018, 10, 483. [CrossRef]

5. Gottesman, M.M. Mechanisms of cancer drug resistance. Annu. Rev. Med. 2002, 53, 615-627. [CrossRef]

6. Ueda, K.; Cardarelli, C.; Gottesman, M.M.; Pastan, I. Expression of a full-length cDNA for the human "MDR1" gene confers resistance to colchicine, doxorubicin, and vinblastine. Proc. Natl. Acad. Sci. USA 1987, 84, 3004-3008. [CrossRef]

7. Higgins, C.F. ABC transporters: From microorganisms to man. Annu. Rev. Cell Biol. 1992, 8, 67-113. [CrossRef]

8. Chan, H.S.; Grogan, T.M.; Haddad, G.; DeBoer, G.; Ling, V. P-glycoprotein expression: Critical determinant in the response to osteosarcoma chemotherapy. J. Natl. Cancer Inst. 1997, 89, 1706-1715. [CrossRef]

9. Ota, E.; Abe, Y.; Oshika, Y.; Ozeki, Y.; Iwasaki, M.; Inoue, H.; Yamazaki, H.; Ueyama, Y.; Takagi, K.; Ogata, T.; et al. Expression of the multidrug resistance-associated protein (MRP) gene in non-small-cell lung cancer. $\mathrm{Br}$. J. Cancer 1995, 72, 550-554. [CrossRef]

10. Bordow, S.B.; Haber, M.; Madafiglio, J.; Cheung, B.; Marshall, G.M.; Norris, M.D. Expression of the multidrug resistance-associated protein (MRP) gene correlates with amplification and overexpression of the N-myc oncogene in childhood neuroblastoma. Cancer Res. 1994, 54, 5036-5040.

11. Callaghan, R.; Luk, F.; Bebawy, M. Inhibition of the multidrug resistance P-glycoprotein: Time for a change of strategy? Drug Metab. Dispos. Biol. Fate Chem. 2014, 42, 623-631. [CrossRef]

12. Zaal, E.A.; Berkers, C.R. The Influence of Metabolism on Drug Response in Cancer. Front. Oncol. 2018, 8, 500. [CrossRef]

13. Iwamoto, H.; Abe, M.; Yang, Y.; Cui, D.; Seki, T.; Nakamura, M.; Hosaka, K.; Lim, S.; Wu, J.; He, X.; et al. Cancer Lipid Metabolism Confers Antiangiogenic Drug Resistance. Cell Metab. 2018, 28, 104-117.e105. [CrossRef]

14. Staubert, C.; Bhuiyan, H.; Lindahl, A.; Broom, O.J.; Zhu, Y.; Islam, S.; Linnarsson, S.; Lehtio, J.; Nordstrom, A. Rewired metabolism in drug-resistant leukemia cells: A metabolic switch hallmarked by reduced dependence on exogenous glutamine. J. Biol. Chem. 2015, 290, 8348-8359. [CrossRef]

15. Staubert, C.; Krakowsky, R.; Bhuiyan, H.; Witek, B.; Lindahl, A.; Broom, O.; Nordstrom, A. Increased lanosterol turnover: A metabolic burden for daunorubicin-resistant leukemia cells. Med. Oncol. 2016, 33, 6. [CrossRef]

16. Hanahan, D.; Weinberg, R.A. Hallmarks of cancer: The next generation. Cell 2011, 144, 646-674. [CrossRef]

17. DeBerardinis, R.J.; Lum, J.J.; Hatzivassiliou, G.; Thompson, C.B. The biology of cancer: Metabolic reprogramming fuels cell growth and proliferation. Cell Metab. 2008, 7, 11-20. [CrossRef]

18. Wise, D.R.; Thompson, C.B. Glutamine addiction: A new therapeutic target in cancer. Trends Biochem. Sci. 2010, 35, 427-433. [CrossRef]

19. Daye, D.; Wellen, K.E. Metabolic reprogramming in cancer: Unraveling the role of glutamine in tumorigenesis. Semin. Cell Dev. Biol. 2012, 23, 362-369. [CrossRef]

20. Eelen, G.; Dubois, C.; Cantelmo, A.R.; Goveia, J.; Bruning, U.; DeRan, M.; Jarugumilli, G.; van Rijssel, J.; Saladino, G.; Comitani, F.; et al. Role of glutamine synthetase in angiogenesis beyond glutamine synthesis. Nature 2018, 561, 63-69. [CrossRef]

21. Long, J.; Lang, Z.W.; Wang, H.G.; Wang, T.L.; Wang, B.E.; Liu, S.Q. Glutamine synthetase as an early marker for hepatocellular carcinoma based on proteomic analysis of resected small hepatocellular carcinomas. Hepatobil. Pancreat. Dis. Int. HBPD Int. 2010, 9, 296-305. [PubMed] 
22. Wang, Y.; Fan, S.; Lu, J.; Zhang, Z.; Wu, D.; Wu, Z.; Zheng, Y. GLUL Promotes Cell Proliferation in Breast Cancer. J. Cell. Biochem. 2017, 118, 2018-2025. [CrossRef] [PubMed]

23. Rosati, A.; Poliani, P.L.; Todeschini, A.; Cominelli, M.; Medicina, D.; Cenzato, M.; Simoncini, E.L.; Magrini, S.M.; Buglione, M.; Grisanti, S.; et al. Glutamine synthetase expression as a valuable marker of epilepsy and longer survival in newly diagnosed glioblastoma multiforme. Neuro-oncology 2013, 15, 618-625. [CrossRef] [PubMed]

24. Fan, S.; Wang, Y.; Zhang, Z.; Lu, J.; Wu, Z.; Shan, Q.; Sun, C.; Wu, D.; Li, M.; Sheng, N.; et al. High expression of glutamate-ammonia ligase is associated with unfavorable prognosis in patients with ovarian cancer. J. Cell. Biochem. 2018, 119, 6008-6015. [CrossRef]

25. Easlon, E.; Tsang, F.; Skinner, C.; Wang, C.; Lin, S.J. The malate-aspartate NADH shuttle components are novel metabolic longevity regulators required for calorie restriction-mediated life span extension in yeast. Genes Dev. 2008, 22, 931-944. [CrossRef]

26. Greenhouse, W.V.; Lehninger, A.L. Occurrence of the malate-aspartate shuttle in various tumor types. Cancer Res. 1976, 36, 1392-1396.

27. Kaufmann, S.H.; Desnoyers, S.; Ottaviano, Y.; Davidson, N.E.; Poirier, G.G. Specific proteolytic cleavage of poly(ADP-ribose) polymerase: An early marker of chemotherapy-induced apoptosis. Cancer Res. 1993, 53, 3976-3985.

28. D'Amours, D.; Sallmann, F.R.; Dixit, V.M.; Poirier, G.G. Gain-of-function of poly(ADP-ribose) polymerase-1 upon cleavage by apoptotic proteases: Implications for apoptosis. J. Cell Sci. 2001, 114, 3771-3778.

29. Wick, A.N.; Drury, D.R.; Nakada, H.I.; Wolfe, J.B. Localization of the primary metabolic block produced by 2-deoxyglucose. J. Biol. Chem. 1957, 224, 963-969.

30. Vila, J.; Thomasset, N.; Navarro, C.; Dore, J.F. In vitro and in vivo anti-tumor activity of L-glutamic acid gamma-monohydroxamate against L1210 leukemia and B16 melanoma. Int. J. Cancer 1990, 45, 737-743. [CrossRef]

31. Thomasset, N.; Goetsch, L.; Hamedi-Sangsari, F.; Tournaire, R.; Malley, S.; Navarro, C.; Dore, J.F.; Vila, J. Inhibition of malate-aspartate shuttle by the antitumor drug L-glutamic acid gamma-monohydroxamate in L1210 leukemia cells. Int. J. Cancer 1992, 51, 329-332. [CrossRef] [PubMed]

32. Thornburg, J.M.; Nelson, K.K.; Clem, B.F.; Lane, A.N.; Arumugam, S.; Simmons, A.; Eaton, J.W.; Telang, S.; Chesney, J. Targeting aspartate aminotransferase in breast cancer. Breast Cancer Res. BCR 2008, 10, R84. [CrossRef] [PubMed]

33. Muthu, M.; Nordstrom, A. Current Status and Future Prospects of Clinically Exploiting Cancer-specific Metabolism-Why Is Tumor Metabolism Not More Extensively Translated into Clinical Targets and Biomarkers? Int. J. Mol. Sci. 2019, 20, 1835. [CrossRef] [PubMed]

34. Kauppinen, R.A.; Sihra, T.S.; Nicholls, D.G. Aminooxyacetic acid inhibits the malate-aspartate shuttle in isolated nerve terminals and prevents the mitochondria from utilizing glycolytic substrates. Biochim. Biophys. Acta 1987, 930, 173-178. [CrossRef]

35. Cluntun, A.A.; Lukey, M.J.; Cerione, R.A.; Locasale, J.W. Glutamine Metabolism in Cancer: Understanding the Heterogeneity. Trends Cancer 2017, 3, 169-180. [CrossRef]

36. Yuneva, M.O.; Fan, T.W.; Allen, T.D.; Higashi, R.M.; Ferraris, D.V.; Tsukamoto, T.; Mates, J.M.; Alonso, F.J.; Wang, C.; Seo, Y.; et al. The metabolic profile of tumors depends on both the responsible genetic lesion and tissue type. Cell Metab. 2012, 15, 157-170. [CrossRef]

37. Davidson, S.M.; Papagiannakopoulos, T.; Olenchock, B.A.; Heyman, J.E.; Keibler, M.A.; Luengo, A.; Bauer, M.R.; Jha, A.K.; O’Brien, J.P.; Pierce, K.A.; et al. Environment Impacts the Metabolic Dependencies of Ras-Driven Non-Small Cell Lung Cancer. Cell Metab. 2016, 23, 517-528. [CrossRef]

38. Lanczky, A.; Nagy, A.; Bottai, G.; Munkacsy, G.; Szabo, A.; Santarpia, L.; Gyorffy, B. miRpower: A web-tool to validate survival-associated miRNAs utilizing expression data from 2178 breast cancer patients. Breast Cancer Res. Treat. 2016, 160, 439-446. [CrossRef]

39. Jurikova, M.; Danihel, L.; Polak, S.; Varga, I. Ki67, PCNA, and MCM proteins: Markers of proliferation in the diagnosis of breast cancer. Acta Histochem. 2016, 118, 544-552. [CrossRef]

40. Safer, B.; Smith, C.M.; Williamson, J.R. Control of the transport of reducing equivalents across the mitochondrial membrane in perfused rat heart. J. Mol. Cell. Cardiol. 1971, 2, 111-124. [CrossRef]

41. Safer, B. The Metabolic Significance of the Malate-Aspartate Cycle in Heart. Circ. Res. 1975, 37, 527-533. [CrossRef] [PubMed] 
42. Rohrig, F.; Schulze, A. The multifaceted roles of fatty acid synthesis in cancer. Nat. Rev. Cancer 2016, 16, 732-749. [CrossRef] [PubMed]

43. Pick, A.M.; Nystrom, K.K. Pazopanib for the treatment of metastatic renal cell carcinoma. Clin. Ther. 2012, 34, 511-520. [CrossRef] [PubMed]

44. Wang, L.; Peng, W.; Wu, T.; Deng, P.; Zhao, Y.L. Increased glutamine anabolism sensitizes non-small cell lung cancer to gefitinib treatment. Cell Death Discov. 2018, 4, 24. [CrossRef]

45. Schoffski, P. Pazopanib in the treatment of soft tissue sarcoma. Expert Rev. Anticancer Ther. 2012, 12, 711-723. [CrossRef]

46. Yvon, A.M.; Wadsworth, P.; Jordan, M.A. Taxol suppresses dynamics of individual microtubules in living human tumor cells. Mol. Biol. Cell 1999, 10, 947-959. [CrossRef]

47. Pruefer, F.G.; Lizarraga, F.; Maldonado, V.; Melendez-Zajgla, J. Participation of Omi Htra2 serine-protease activity in the apoptosis induced by cisplatin on SW480 colon cancer cells. J. Chemother. (Florence Italy) 2008, 20, 348-354. [CrossRef]

48. Sanjana, N.E.; Shalem, O.; Zhang, F. Improved vectors and genome-wide libraries for CRISPR screening. Nat. Methods 2014, 11, 783-784. [CrossRef]

49. Shalem, O.; Sanjana, N.E.; Hartenian, E.; Shi, X.; Scott, D.A.; Mikkelson, T.; Heckl, D.; Ebert, B.L.; Root, D.E.; Doench, J.G.; et al. Genome-scale CRISPR-Cas9 knockout screening in human cells. Science 2014, 343, 84-87. [CrossRef]

(C) 2019 by the authors. Licensee MDPI, Basel, Switzerland. This article is an open access article distributed under the terms and conditions of the Creative Commons Attribution (CC BY) license (http://creativecommons.org/licenses/by/4.0/). 